\title{
Optimising Graphene Visibility in van der Waals Heterostructures
}

\author{
Thanmay S. Menon ${ }^{1, *}$, Simli Mishra ${ }^{2}$, Vidhu Catherine Antony ${ }^{3}$, \\ Kiranmayi Dixit ${ }^{4, \dagger}$, Saloni Kakkar ${ }^{5}$, Tanweer Ahmed ${ }^{5}$, Saurav Islam ${ }^{5}$, \\ Aditya Jayaraman ${ }^{5}$, Kimberly Hsieh ${ }^{5, *}$, Paritosh Karnatak ${ }^{5, \ddagger, *}$ and Arindam Ghosh ${ }^{5,6}$ \\ 1 Undergraduate Programme, Indian Institute of Science, Bangalore 560012, India \\ 2 Department of Physical Sciences, \\ Indian Institute of Science Education and Research (IISER) Kolkata, \\ Mohanpur 741246, Nadia, West Bengal, India \\ 3 Department of Physics, Bangalore University, Bangalore 560 056, India \\ 4 Department of Physics, Indian Institute of \\ Technology Guwahati, Guwahati 781 039, Assam, India \\ 5 Department of Physics, Indian Institute of Science, Bangalore 560012, India and \\ 6 Centre for Nano Science and Engineering, \\ Indian Institute of Science, Bangalore 560012, Indid*
}

\begin{abstract}
Graphene constitutes one of the key elements in many functional van der Waals heterostructures. However, it has negligible optical visibility due to its monolayer nature. Here we study the visibility of graphene in various van der Waals heterostructures and include the effects of the source spectrum, oblique incidence and the spectral sensitivity of the detector to obtain a realistic model. A visibility experiment is performed at different wavelengths, resulting in a very good agreement with our calculations. This allows us to reliably predict the conditions for better visibility of graphene in van der Waals heterostructures. The framework and the codes provided in this work can be extended to study the visibility of any $2 \mathrm{D}$ material within an arbitrary van der Waals heterostructure.
\end{abstract}

\footnotetext{
* These authors contributed equally to this work. Correspondence should be addressed to K.H. (kimberly@iisc.ac.in) or P.K. (paritosh@iisc.ac.in).;

$\dagger$ Current address: Department of Physics and Astronomy, Purdue University, West Lafayette, IN 47907, USA;

$\ddagger$ Current address: Department of Physics, University of Basel, Klingelbergstrasse 82, CH-4056 Basel, Switzerland
} 


\section{INTRODUCTION}

The family of van der Waals materials has now expanded beyond graphene and offers a wide range of material functionalities, such as semiconductors ${ }^{12}$, insulators ${ }^{3 / 5}$, superconductors ${ }^{6 / 7}$ and ferromagnets $\frac{89}{}$. In addition, van der Waals heterostructures are fabricated by stacking individual 2D materials to obtain compound materials with novel functionalities 10 12. However, graphene is often difficult to locate through an optical microscope during or after the heterostructure assembly due to its near-optical transparency. While the visibility of graphene on $\mathrm{SiO}_{2} / \mathrm{Si}$ substrate has been studied before ${ }^{13}$, its visibility in various configurations of van der Waals heterostructures has not yet been explored. In this paper, we develop a stepwise, robust formalism to study the visibility of graphene in various heterostructures and include practical considerations such as the details of the source spectrum, oblique incidence which is necessary at high magnifications $\sqrt{15 \mid 18} \sqrt[23]{2}$, and the spectral sensitivity of the detector. We perform the visibility calculations for graphene-BN, graphene-BN-MoS 2 , graphene- $\mathrm{MoS}_{2}$-BN and graphene- $\mathrm{MoS}_{2}$ heterostructures on $\mathrm{SiO}_{2}$ substrate and suggest conditions for better visibility. In order to corroborate our theoretical calculations, we experimentally determine the optical visibility of a graphene-BN-MoS $\mathrm{S}_{2}$ and graphene- $\mathrm{MoS}_{2}$ heterostructures at different wavelengths. Our methods, codes and table of information can be employed to study the visibility of most $2 \mathrm{D}$ van der Waals materials in an arbitrary heterostructure configuration.

\section{METHODS}

For the theoretical calculations, a thin film interference model was assumed where the reflection coefficients were calculated using Fresnel's equations. Reflection coefficients $r_{i, s}$ and $r_{i, p}$ (for $s$ and $p$ polarisations respectively) for the interface between $i^{\text {th }}$ and $i+1^{\text {th }}$ layer (see Fig. $\mathrm{S1}$ b) can be written as ${ }^{24 \mid 25}$ :

$$
\begin{array}{r}
r_{i, s}=\frac{\tilde{n}_{i} \cos \left(\theta_{i}\right)-\tilde{n}_{i+1} \cos \left(\theta_{i+1}\right)}{\tilde{n}_{i} \cos \left(\theta_{i}\right)+\tilde{n}_{i+1} \cos \left(\theta_{i+1}\right)} \\
r_{i, p}=\frac{\tilde{n}_{i+1} \cos \left(\theta_{i}\right)-\tilde{n}_{i} \cos \left(\theta_{i+1}\right)}{\tilde{n}_{i+1} \cos \left(\theta_{i}\right)+\tilde{n}_{i} \cos \left(\theta_{i+1}\right)}
\end{array}
$$

where $\tilde{n}_{i}\left(\tilde{n}_{i+1}\right)$ and $\theta_{i}\left(\theta_{i+1}\right)$ corresponds to the refractive index and angle from the normal 
respectively in the $i^{\text {th }}\left(i+1^{\text {th }}\right)$ layer. $\theta_{i}$ can be obtained using Snell's law applied to the $i^{\text {th }}$ layer and is complex for an absorbent material.

Often one or more layers in the stack are optically anisotropic, i.e., the in-plane and outof-plane polarisation refractive indices are not equal. For uniaxial crystals, the calculation of $r_{i, s}$ remains trivial. However, the refractive index in the case of $p$-polarisation is obtained by solving the following self-consistent equation ${ }^{25}$ in $\theta$ :

$$
\frac{1}{\tilde{n}_{p}^{2}}=\frac{\cos ^{2} \theta}{\tilde{n}_{\|}^{2}}+\frac{\sin ^{2} \theta}{\tilde{n}_{\perp}^{2}}
$$

where $n_{\|}$and $n_{\perp}$ are the in-plane and out-of-plane polarisation refractive indices respectively.

After calculating $r_{i}$ (for $s$ - and $p$ polarisations), the reflectivity can be obtained from the N-layer reflection formula, $\tilde{R}_{N}$ which is obtained recursively as follows 2627 :

If $\phi_{i}=4 \pi \tilde{n}_{i} \cos \theta_{i} d_{i} / \lambda$ is the phase shift due to the optical path difference in the $i^{\text {th }}$ layer of thickness $d_{i}$,

$$
\tilde{R}_{N}=R_{N} e^{i \delta_{N}}=\frac{r_{0}+\tilde{R}_{N-1} e^{-i \phi_{0}}}{1+r_{0} \tilde{R}_{N-1} e^{-i \phi_{0}}}
$$

where $\delta_{N}$ is the total phase acquired after transmission through $N$ layers, $r_{0}$ is the reflection coefficient of the topmost interface (see Fig. S1b) and is given by (1) or (2) depending on the polarisation of incident light, and $\tilde{R}_{N-1}$ is the reflection from $N-1$ layers which is computed by applying (4) to the substrate and using $r_{1}$ and $\phi_{1}$ instead of $r_{0}$ and $\phi_{0}$. This method is repeated for subsequent layers till we reach $\tilde{R}_{0}=r_{N}$ which is the reflection from the interface between the Nth layer and semi-infinite medium of silicon. The reflected intensity from the entire stack is given by $I=\left|\tilde{R}_{N}\right|^{2}$.

Accounting for oblique incidence is especially important when viewing the samples at large magnifications (especially $100 \times$ ) because of the high numerical aperture $\left(\alpha_{N A}\right)$ of the objective. To take this into account, we assume that the incident beam has a gaussian profile ${ }^{21}$ and integrate the reflected intensity for both polarisations over angles from 0 to $\theta_{M}=a \sin \left(\alpha_{N A}\right)$

$$
I(\lambda)=\int_{0}^{\theta_{m}} I(\theta, \lambda) e^{-\frac{2 \rho^{2}}{\rho_{m}^{2}}} \tan \theta d \theta
$$


where $\rho=\tan \theta$ and $\rho_{m}=\tan \theta_{m}$ (see Fig. S1b)

In the visibility calculation, the normalisation constants for the gaussian distribution cancel out eventually. As the incident beam is unpolarised, the reflected intensity is the average of the reflected intensities due to $s$ and $p$ polarisations.

The visibility (also known as Michelson's contrast) ${ }^{28 \mid 29}$ is defined as:

$$
\operatorname{Visibility~}(\text { in } \%)=100 \times \frac{I_{s}-I_{g}}{I_{s}+I_{g}}
$$

$I_{s}$ corresponds to the reflection from the substrate and $I_{g}$ refers to the reflection from the entire heterostucture. Positive or negative value of visibility corresponds to graphene appearing darker or lighter than the substrate, respectively.

Often the spectrum of the source and the spectral sensitivity of the detector must also be included to obtain a more accurate value of the visibility. For a typical RGB digital camera, if the red, green and blue channel spectral sensitivities are $\omega_{R}(\lambda), \omega_{G}(\lambda)$ and $\omega_{B}(\lambda)$ and the spectrum of the source is $S(\lambda)$, then the reflected intensities picked up by the red, green and blue channels are $20 \mid 30$ :

$$
I_{j}=\int_{0}^{\infty} I(\lambda) \omega_{j}(\lambda) S(\lambda) d \lambda
$$

where $\mathrm{j}=\mathrm{R}, \mathrm{G}, \mathrm{B}$.

By substituting these intensities in the visibility formula (6), one can calculate the visibility for each colour channel. One should also include the spectral dependence of lenses, mirrors, etc. for more sensitive applications.

The dependence of graphene's visibility on wavelength for different angles of incidence is shown in Figs. $\mathrm{S} 1 \mathrm{k}$ and $\mathrm{S} 1 \mathrm{~d}$ for graphene- $\mathrm{SiO}_{2}$ and graphene- $\mathrm{BN}-\mathrm{SiO}_{2}$ heterostructures respectively. Although there is a shift in the peak position for both heterostructures, the relative shifts in the height of the peaks are different for both heterostructure configurations. Specifically, comparing between incidence at $60^{\circ}$ and normal incidence, there is a greater difference in the height of the peaks for graphene- $\mathrm{BN}-\mathrm{SiO}_{2}$ than for graphene- $\mathrm{SiO}_{2}$. This explains why accounting for oblique incidence is important for complicated heterostructure configurations. The codes employed in this work are available on GitHub 31 .

The heterostructures presented in this work were prepared by mechanically exfoliating the individual flakes of graphene, $\mathrm{BN}$ and $\mathrm{MoS}_{2}$ on separate $\mathrm{SiO}_{2}$ substrates followed by micro- 
mechanical transfer technique using a polydimethylsiloxane (PDMS) stamp spin-coated with a transparent sacrificial polymer layer ${ }^{\sqrt[32]{34}}$ Similarly prepared graphene-based heterostructures have been previously shown to exhibit relatively high mobilities when encapsulated by $\mathrm{BN}^{\sqrt{34135}}$ and have also found widespread functionality as ultra-sensitive photodetectors due to their efficient interlayer charge transfer ${ }^{33336}$.

We have analysed and performed visibility calculations for three heterostructure configurations: graphene- $\mathrm{BN}-\mathrm{SiO}_{2}$ and graphene- $\mathrm{MoS}_{2}-\mathrm{SiO}_{2}$, and graphene- $\mathrm{BN}-\mathrm{MoS}_{2}-\mathrm{SiO}_{2}$. The images were taken using an Olympus UC30 camera mounted on an Olympus BX51 microscope through a $100 \times$ objective. The heterostructures were illuminated using standard light emitting diodes (LEDs) of different wavelengths at a constant power of $2 \mathrm{~mW}$ and the exposure time (1-2 sec) was set so as not to saturate any of the channels but also yield sufficient signal-to-noise ratio.The images were split into three RGB channels and were analysed using ImageJ software. The relative intensities of these channels depend on the particular LED being used. The channel with the maximum signal-to-noise ratio for each LED was used in the calculations. To minimise errors due to uneven illumination, $I_{s}$ was recorded at a point on the substrate close to the point on graphene where $I_{g}$ was recorded and then visibility was calculated using (6). This was done multiple times over the whole sample for the same LED and the average visibility is plotted. The spectrum of the LEDs and the spectral sensitivity of the camera (see supplementary information Fig. S1) were incorporated in the calculations using (7). The experiment was performed using standard LEDs rather than the microscope's incandescent light source since it provides a better control over the choice and range of the source wavelength and also avoids complications that arise from digital image processing of a white-light image (such as white-balance), unique to each camera and its imaging software. The thickness of BN used in the stack was determined to be $11 \mathrm{~nm}$ using atomic force microscopy (AFM) and Raman spectroscopy was used to verify that $\mathrm{MoS}_{2}$ and graphene were monolayers. We used a standard $\mathrm{SiO}_{2}$-Si substrate with an $\mathrm{SiO}_{2}$ thickness of $285 \mathrm{~nm}$. We have used both the in-plane and out-of-plane refractive indices of graphene ${ }^{37}$ and $\mathrm{MoS}_{2}{ }^{38}$ and the refractive indices of $\mathrm{BN}^{399}$ and $\mathrm{SiO}_{2}{ }^{40}$ (both of which have zero extinction coefficients) as well as that of $\mathrm{Si}$ i1 . 


\section{RESULTS AND DISCUSSION}

Figs. S2(a) and S2(c) show the experimental results along with the theoretical calculations for two-layer graphene-BN and graphene- $\mathrm{MoS}_{2}$ heterostructures with $\mathrm{SiO}_{2}$ as substrate. The respective thicknesses were fixed at the experimentally determined values. To the right of Figs. S2(a) and S2(c) are the microscope images of the respective heterostructures illuminated with LEDs of different wavelengths. Although we have performed the visibility experiment using seven LEDs with wavelengths spread over the visible regime, we have shown only selected images in those colour channels with maximum signal-to-noise ratio. $\alpha_{N A}$ obtained from the best fit of the visibilities was $\sim 0.88$ which is very close to the value of 0.9 provided by Olympus. We can see very close agreement between theory and experiment, which suggests that our model for computing visibilities is sufficiently accurate. Minor deviations of the experimental values from the theoretical values may be due to the incident beam not being strictly gaussian in nature. In Figs. S2b and $\mathrm{S} 2 \mathrm{~d}$, we have presented the results of our visibility calculations for the graphene- $\mathrm{BN}$ and graphene-MoS $\mathrm{S}_{2}$ heterostructures as a function of varying substrate and underlayer thicknesses. It must be mentioned here that the calculations in Figs. S2(a) and S2 (c) incorporated the spectrum of the LEDs in order to corroborate the results of the visibility experiment while the calculations in Figs. $\mathrm{S} 2 \mathrm{~b}$ and $\mathrm{S} 2 \mathrm{~d}$ have been performed assuming a typical $\alpha_{N A}$ of 0.9 and integrating over the spectrum of an incandescent light source of blackbody temperature $3100 \mathrm{~K}$, the most common light source used in optical microscopes employed for the searching of suitable flakes of van der Waal materials for device fabrication. Although we have assumed the spectral sensitivity of our camera (SONY ICX252AQ CCD image sensor) in our calculations, it does not differ widely across different cameras ${ }^{42}$. Such a model gives us three values of visibility (one for each colour channel). In these figures, we have plotted the maximum of the absolute values of the three visibilities arising from red, green and blue channels. From our experiments, we have arrived at an absolute visibility threshold value of $2.5 \%$ above which the graphene would be visible. In this way, the phase space of $\mathrm{BN}_{-} \mathrm{SiO}_{2}$ thicknesses in Fig. S2b can be divided into 'islands of visibility' (bounded by dashed white lines in the figure), where graphene would be visible if the $\mathrm{BN}$ and $\mathrm{SiO}_{2}$ thicknesses lie within such an island. The same has been done in Fig. $\mathrm{S} 2 \mathrm{~d}$ for graphene-MoS $\mathrm{M}_{2}$ heterostructure. In these figures, we see that using lower thicknesses of $\mathrm{SiO}_{2}$ substrate results in higher visibility for graphene 
in both graphene-BN and graphene- $\mathrm{MoS}_{2}$ heterostructures. Fig. $\mathrm{S} 2 \mathrm{~b}$ also indicates that in a graphene-BN heterostructure on $\mathrm{SiO}_{2}$ (with a typical thickness of $300 \mathrm{~nm}$ ), the visibility of graphene reduces with increasing BN thickness. For BN thicknesses above $\approx 42$ $\mathrm{nm}$, graphene is visible only at low $\mathrm{SiO}_{2}$ thicknesses and in the supplementary information (Fig. S2), we have shown that a $380 \mathrm{~nm}$ double layer of polymethyl methacrylate (PMMA) spin-coated on the heterostructure improves the visibility significantly for thicker BN on 285 $\mathrm{nm} \mathrm{SiO}_{2}$.

Fig. 53 a shows the experimental results and theoretical calculations for graphene-BN$\mathrm{MoS}_{2}$ heterostructure (the right panels show the corresponding optical images under the illumination of various LEDs). We see good agreement between the theory and experiment even in a three-layer heterostructure. The insets in Figs. $S 2 a, S 2 c$ and $S 3 a$ show that a model that considers only normal incidence is inadequate in explaining the experimental results and therefore, one must resort to an oblique incidence model to make reliable predictions. Figs. S3b and S3. show visibility calculations for graphene-BN-MoS 2 and graphene- $\mathrm{MoS}_{2^{-}}$ $\mathrm{BN}$ heterostructures as a function of $\mathrm{SiO}_{2}$ and $\mathrm{BN}$ thicknesses and assuming $\mathrm{MoS}_{2}$ to be a monolayer. We observe that this figure looks similar to the graphene-BN graph. This is because of the similarity between the refractive indices of $\mathrm{MoS}_{2}$ and $\mathrm{BN}$ especially in the red and green wavelength ranges and because we are only considering monolayer $\mathrm{MoS}_{2}$. Similar to the graphene-BN heterostructure, lower $\mathrm{SiO}_{2}$ thickness is recommended for better visibility of graphene.

Fig. S4 shows the maximum absolute visibility for various heterostructure configurations on $285 \mathrm{~nm}$ of $\mathrm{SiO}_{2}$ along with the peak wavelengths. It also suggests various thicknesses of van der Waals materials that can be used for viewing graphene on $285 \mathrm{~nm} \mathrm{SiO}_{2}$. These values tend to lie in the green wavelengths which our eyes are most sensitive to. This is not a coincidence but a result of choosing $\mathrm{SiO}_{2}$ thickness to be $285 \mathrm{~nm}$. From our calculations, we can conclude that effects of van der Waals materials under graphene are fundamentally no different when compared to a regular dielectric. Although the effect of the substrate dominates over non-metallic van der Waals materials, using an $\mathrm{SiO}_{2}$ substrate of an arbitrary thickness to improve the visibility is often neither desirable nor practical. Instead, our aim in this paper is to provide a general framework and codes to allow the readers to calculate the visibility of any crystal within a heterostructure. In case of poor visibility, the readers may consider choosing an underlayer (say, BN) of appropriate thickness, if the calculations 
predict that this improves the visibility. From the figure, we also note that graphene- $\mathrm{Bi}_{2} \mathrm{Se}_{3}$ has very poor visibility. This is attributed to the high extinction coefficient of $\mathrm{Bi}_{2} \mathrm{Se}_{3} 43$, due to the existence of its metallic surface states. Hence, we expect graphene's visibility to be adversely affected by metallic single- to few-layers. A similar reduction in contrast was already observed for graphene on gold substrates 22123 .

In the supplementary information (Fig. S2), we have computed the visibility for other common heterostructure configurations such as BN-graphene-BN, PMMA-graphene-BN, PMMA-graphene- $\mathrm{MoS}_{2}$ and a recently proposed photocatalyst, graphene-ZrS ${ }_{2}{ }^{44}$ using the same method and have identified optimal thicknesses of different layers for maximum visibility.

In conclusion, we have studied the conditions for the optimal visibility of graphene in van der Waals heterostructures. We have also performed experiments to demonstrate the accuracy of our predictions. Our methods and codes may be directly employed to calculate the visibility of a van der Waals material in a heterostructure.

\section{Acknowledgements}

The authors thank Am. Ghosh for use of a spectrometer and SERC, IISc for their computational facilities. The authors also acknowledge G. Singh and S. Majumder for useful discussions.

\section{REFERENCES}

[1] T. Paul, S. Ghatak, and A. Ghosh, Nanotechnology 27, 125706 (2016).

[2] X. Duan, C. Wang, A. Pan, R. Yu, and X. Duan, Chem. Soc. Rev. 44, 8859 (2015),

[3] S. Bhattacharjee, K. L. Ganapathi, H. Chandrasekar, T. Paul, S. Mohan, A. Ghosh, S. Raghavan, and N. Bhat, Adv. Electron. Mater. 3, 1600358 (2017).

[4] L. Britnell, R. V. Gorbachev, R. Jalil, B. D. Belle, F. Schedin, M. I. Katsnelson, L. Eaves, S. V. Morozov, A. S. Mayorov, N. M. R. Peres, A. H. Castro Neto, J. Leist, A. K. Geim, L. A. Ponomarenko, and K. S. Novoselov, Nano Lett. 12, 1707 (2012).

[5] D. L. Duong, S. J. Yun, and Y. H. Lee, ACS Nano 11, 11803 (2017). 
[6] A. F. Kusmartseva, B. Sipos, H. Berger, L. Forró, and E. Tutiš, Phys. Rev. Lett. 103, 236401 (2009).

[7] X. Xi, L. Zhao, Z. Wang, H. Berger, L. Forró, J. Shan, and K. F. Mak, Nat. Nanotechnol. 10, $765(2015)$.

[8] B. Huang, G. Clark, E. Navarro-Moratalla, D. R. Klein, R. Cheng, K. L. Seyler, D. Zhong, E. Schmidgall, M. A. McGuire, D. H. Cobden, W. Yao, D. Xiao, P. Jarillo-Herrero, and X. Xu, Nature 546, 270 (2017)

[9] C. Gong, L. Li, Z. Li, H. Ji, A. Stern, Y. Xia, T. Cao, W. Bao, C. Wang, Y. Wang, Z. Q. Qiu, R. J. Cava, S. G. Louie, J. Xia, and X. Zhang, Nature 546, 265 (2017).

[10] Y. Liu, N. O. Weiss, X. Duan, H.-C. Cheng, Y. Huang, and X. Duan, Nat. Rev. Mater. 1, 16042 (2016).

[11] T. Georgiou, R. Jalil, B. D. Belle, L. Britnell, R. V. Gorbachev, S. V. Morozov, Y.-J. Kim, A. Gholinia, S. J. Haigh, O. Makarovsky, L. Eaves, L. A. Ponomarenko, A. K. Geim, K. S. Novoselov, and A. Mishchenko, Nat. Nanotechnol. 8, 100 (2013).

[12] K. Roy, M. Padmanabhan, S. Goswami, T. P. Sai, S. Kaushal, and A. Ghosh, Solid State Commun. 175-176, 35 (2013).

[13] S. Roddaro, P. Pingue, V. Piazza, V. Pellegrini, and F. Beltram, Nano Lett. 7, 2707 (2007).

[14] P. Blake, E. W. Hill, A. H. Castro Neto, K. S. Novoselov, D. Jiang, R. Yang, T. J. Booth, and A. K. Geim, Appl. Phys. Lett. 91, 063124 (2007).

[15] C. Casiraghi, A. Hartschuh, E. Lidorikis, H. Qian, H. Harutyunyan, T. Gokus, K. S. Novoselov, and A. C. Ferrari, Nano Lett. 7, 2711 (2007).

[16] Z. H. Ni, H. M. Wang, J. Kasim, H. M. Fan, T. Yu, Y. H. Wu, Y. P. Feng, and Z. X. Shen, Nano Lett. 7, 2758 (2007).

[17] H. Li, J. Wu, X. Huang, G. Lu, J. Yang, X. Lu, Q. Xiong, and H. Zhang, ACS Nano 7, 10344 (2013).

[18] D. Bing, Y. Wang, J. Bai, R. Du, G. Wu, and L. Liu, Opt. Commun. 406, 128 (2018).

[19] F. Huang, J. Phys. Chem. C (2019).

[20] B. S. Jessen, P. R. Whelan, D. M. Mackenzie, B. Luo, J. D. Thomsen, L. Gammelgaard, T. J. Booth, and P. Bøggild, Sci. Rep. 8, 2 (2018).

[21] M. Bruna and S. Borini, J. Phys. D Appl. Phys. 42, 175307 (2009). 
[22] J. M. Katzen, M. Velickỳ, Y. Huang, S. Drakeley, W. Hendren, R. M. Bowman, Q. Cai, Y. Chen, L. H. Li, and F. Huang, ACS Applied Materials \& Interfaces 10, 22520 (2018).

[23] M. Velickỳ, W. R. Hendren, G. E. Donnelly, J. M. Katzen, R. M. Bowman, and F. Huang, Nanotechnology 29, 275205 (2018).

[24] E. Hecht, Optics (Pearson, New York, 2016).

[25] M. Born, E. Wolf, A. B. Bhatia, P. C. Clemmow, D. Gabor, A. R. Stokes, A. M. Taylor, P. A. Wayman, and W. L. Wilcock, Principles of Optics (Cambridge University Press, Cambridge, 1999).

[26] H. Anders, Thin Films in Optics (The Focal Press, London, 1965).

[27] Z. Knittl, Optics of Thin Films: An Optical Multilayer Theory (Wiley, London, 1976).

[28] I. Jung, M. Pelton, R. Piner, D. A. Dikin, S. Stankovich, S. Watcharotone, M. Hausner, and R. S. Ruoff, Nano Lett. 7, 3569 (2007)

[29] A. Michelson, Studies in Optics (Dover Publications, New York, 1995).

[30] P. Stigell, K. Miyata, and M. Hauta-Kasari, Pattern Recogn. Image Anal. 17, 233 (2007).

[31] T. Menon, Calculating visibility of van der Waals heterostructures (2019), retrieved from https://github.com/Thanmay-Menon/visibility.

[32] P. J. Zomer, S. P. Dash, N. Tombros, and B. J. van Wees, Appl. Phys. Lett. 99, 232104 $(2011)$.

[33] K. Roy, M. Padmanabhan, S. Goswami, T. P. Sai, G. Ramalingam, S. Raghavan, and A. Ghosh, Nat. Nanotechnol. 8, 826 (2013).

[34] P. Karnatak, T. P. Sai, S. Goswami, S. Ghatak, S. Kaushal, and A. Ghosh, Nat. Commun. 7, 13703 (2016).

[35] M. A. Aamir, P. Karnatak, A. Jayaraman, T. P. Sai, T. Ramakrishnan, R. Sensarma, and A. Ghosh, Phys. Rev. Lett. 121, 136806 (2018).

[36] K. Roy, T. Ahmed, H. Dubey, T. P. Sai, R. Kashid, S. Maliakal, K. Hsieh, S. Shamim, and A. Ghosh, Adv. Mater. 30, 1704412 (2018).

[37] A. B. Djurišić and E. H. Li, J. Appl. Phys. 85, 7404 (1999).

[38] N. N. Hieu, V. V. Ilyasov, T. V. Vu, N. A. Poklonski, H. V. Phuc, L. T. Phuong, B. D. Hoi, and C. V. Nguyen, Superlatt. Microstruct. 115, 10 (2018).

[39] R. V. Gorbachev, I. Riaz, R. R. Nair, R. Jalil, L. Britnell, B. D. Belle, E. W. Hill, K. S. Novoselov, K. Watanabe, T. Taniguchi, A. K. Geim, and P. Blake, Small 7, 465 (2011), 
1008.2868 .

[40] L. V. Rodríguez-de Marcos, J. I. Larruquert, J. A. Méndez, and J. A. Aznárez, Opt. Mater. Express 6, 3622 (2016).

[41] D. E. Aspnes and A. A. Studna, Phys. Rev. B 27, 985 (1983).

[42] J. Jiang, D. Liu, J. Gu, and S. Susstrunk, in Proceedings of IEEE Workshop on Applications of Computer Vision (2013) pp. 168-179.

[43] M. Eddrief, F. Vidal, and B. Gallas, J. Phys. D Appl. Phys. 49, 505304 (2016).

[44] X. Zhang, Z. Meng, D. Rao, Y. Wang, Q. Shi, Y. Liu, H. Wu, K. Deng, H. Liu, and R. Lu, Energy Environ. Sci. 9, 841 (2016). 


\section{Supplementary Information}

\section{TABLE OF REFERENCES FOR REFRACTIVE INDICES OF VARIOUS 2D MATERIALS}

\begin{tabular}{|c|c|c|}
\hline S.I No. & Material & Reference \\
\hline \hline 1 & Graphene & DjuriÂšic et al., J. Appl. Phys. 85, 7404 (1999) \\
\hline 2 & $\mathrm{BN}$ & Gorbachev et al., Small 7, $465(2011)$ \\
\hline 3 & $\mathrm{MoS}_{2}$ & Hieu et al., Superlatt. Microstruct. 115, $10(2018)$ \\
\hline 4 & $\mathrm{Bi}_{2} \mathrm{Se}_{3}$ & Eddrief et al., J Phys. D Appl. Phys. 49, 505304 (2016) \\
\hline 5 & $\mathrm{SiO}_{2}$ & RodrÃguez-de Marcos et al., Opt. Mater. Express 6, 3622 (2016) \\
\hline 6 & $\mathrm{Si}$ & Aspnes et al., Phys. Rev. B 27, 985 (1983) \\
\hline
\end{tabular}

\section{EXPERIMENTAL DETAILS}

The visibility of graphene-BN and graphene-BN-MoS 2 heterostructure stacks were measured on different parts of the same sample. Figs. S1(a) and (b) show the images of graphene-BN-MoS 2 and graphene- $\mathrm{MoS}_{2}$ sample under different light sources. The light sources used were LEDs of different colours and their intensities were calibrated to ensure uniform intensity of the LEDs. We use a gaussian beam approximation for the LEDs since the incident light beam is narrower than the input aperture of the objective lens (some high-end microscope light sources provide uniform illumination). The spectra of the LEDs, recorded using an Ocean optics spectrometer, is given in Fig. S1(e). The images were taken using an Olympus UC30 camera, which was equipped with a SONY ICX 252 AQ CCD image sensor. This sensor's spectral sensitivity (obtained from the sensor's datasheet) is given in Fig. S1(f). These images were then split into RGB channels and the channel with maximum signal-to-noise ratio was analysed using ImageJ software. Figs. S1(c) and (d) show the same sample under an ordinary white light source (blackbody of temperature $3100 \mathrm{~K}$ ). 


\section{RESULTS FOR OTHER HETEROSTRUCTURES}

Figs. S2(a) and S2(b) show the effect of polymethyl methacrylate (PMMA) polymer on the maximum absolute visibility of graphene-BN and graphene- $\mathrm{MoS}_{2}$ heterostructures. A standard double layer of PMMA-495K (A4) followed by PMMA-950K (A4), spin-coated at $\sim 4500 \mathrm{rpm}$, gives a thickness of $\sim 380 \mathrm{~nm}^{1}$. We have assumed the same refractive indices

for both coatings of $\mathrm{PMMA}^{2}$. We see that in case of graphene-BN heterostructure, PMMA increases the range of $\mathrm{BN}$ thicknesses which are optically visible under a microscope, especially at $\sim 300 \mathrm{~nm}$ and $\sim 90 \mathrm{~nm}$ thicknesses of $\mathrm{SiO}_{2}$. However, in the case of graphene- $\mathrm{MoS}_{2}$ heterostructure, at $285 \mathrm{~nm} \mathrm{SiO}_{2}$ thickness, graphene is just barely visible on monolayer $\mathrm{MoS}_{2}$.

Fig. S2(c) shows maximum absolute visibility of BN-graphene-BN heterostructure where the thickness of the top layer of $\mathrm{BN}$ is fixed at $10 \mathrm{~nm}$ and the thickness of the bottom $\mathrm{BN}$ layer below graphene is allowed to vary. This figure shows features very similar to that of graphene-BN heterostructure except that the maximum BN thickness for graphene to be visible is lesser here. Fig S2 (d) shows the maximum absolute visibility map of graphene- $\mathrm{Bi}_{2} \mathrm{Se}_{3}$ heterostructure. Here the maximum absolute visibility is calculated from $10 \mathrm{~nm}$-thick $\mathrm{Bi}_{2} \mathrm{Se}_{3}$ onwards as there is evidence that the refractive index of $\mathrm{Bi}_{2} \mathrm{Se}_{3}$ abruptly changes below $10 \mathrm{~nm}^{3}$. We can see that in the range of thicknesses we have considered for $\mathrm{Bi}_{2} \mathrm{Se}_{3}$, the visibility of graphene is very poor. This is attributed to the high extinction coefficient of $\mathrm{Bi}_{2} \mathrm{Se}_{3}$, due to its metallic surface states.

From Fig. S3a, we can see that the maximum visibility of graphene on monolayer $\mathrm{ZrS}_{2}$ $\left(\mathrm{SiO}_{2}\right.$ thickness being $\left.285 \mathrm{~nm}\right)$ is close to $6 \%$ at wavelength $\lambda \approx 527 \mathrm{~nm}$. This is on par with graphene's visibility on BN which would be very helpful during device fabrication. The refractive index and monolayer thickness of $\mathrm{ZrS}_{2}$ were taken from Ref. ${ }^{4}$ and Ref. ${ }^{5}$ respectively.

\section{REFERENCES}

[1] MicroChem, (2001), retrieved from http://microchem.com/pdf/PMMA_Data_Sheet.pdf.

[2] N. Sultanova, S. Kasarova, and I. Nikolov, Acta Phys. Pol. A 116, 585 (2009).

[3] C. Vajner, H. Yan, L. Guo, M. Mathews, M. Kuhlman, S. Benefield, S. Ulrich, E. Zolghadr, 
P. Kung, L. Li, P. T. Araujo, and H.-T. Wang, 2D Mater. 3, 021010 (2016).

[4] T. V. Vu, A. Lavrentyev, D. V. Thuan, C. V. Nguyen, O. Khyzhun, B. Gabrelian, K. C. Tran, H. L. Luong, P. D. Tung, K. D. Pham, et al., Superlattices Microstruct. 125, 205 (2019).

[5] X. Wang, L. Huang, X.-W. Jiang, Y. Li, Z. Wei, and J. Li, J. Mater. Chem. C 4, 3143 (2016). 
(a)



(b)



(c)



(d)

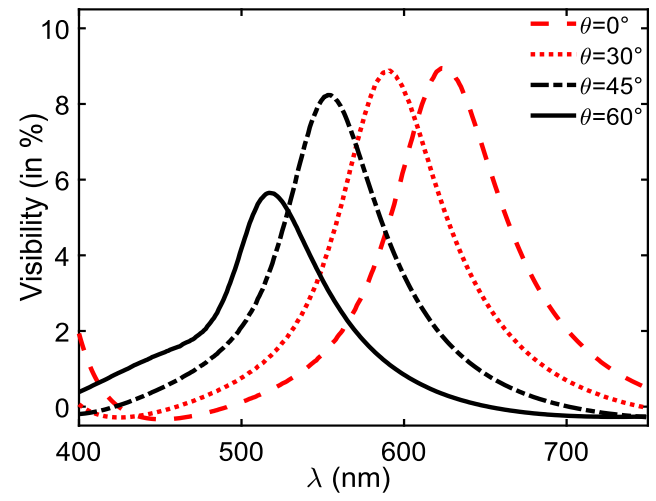

Figure S1. (a) Block diagram showing how visibility is calculated for an arbitrary van der Waals heterostructure (b) A schematic of the $N$-layer stack illuminated by light passing through the objective. (c,d) Plot of visibility vs wavelength of (c) graphene on $\mathrm{SiO}_{2}-\mathrm{Si}$ substrate and (d) graphene on $\mathrm{BN}_{-} \mathrm{SiO}_{2}$-Si substrate for different angles of incidence. 
(a)



(c)





MoS 2 Graphene



(b)



(d)



Figure S2. (a,c) Visibility as a function of wavelength showing experimental and theoretical plots for (a) graphene-BN (c) graphene- $\mathrm{MoS}_{2}$ heterostructures. The panels to the right of (a) and (c) are the optical micrographs of the respective samples under the illumination of LEDs of different wavelengths (namely $465 \mathrm{~nm}, 535 \mathrm{~nm}, 590 \mathrm{~nm}$ and $635 \mathrm{~nm}$ ) converted to greyscale. The scale bar at the bottom right is $2 \mu \mathrm{m}$. Color plots of the maximum of absolute visibilities of the three channels as a function of (b) $\mathrm{SiO}_{2}$ thickness and $\mathrm{BN}$ thickness (d) $\mathrm{SiO}_{2}$ thickness and number of layers of $\mathrm{MoS}_{2}$. White dashed lines are contours drawn at $2.5 \%$ absolute visibility. 


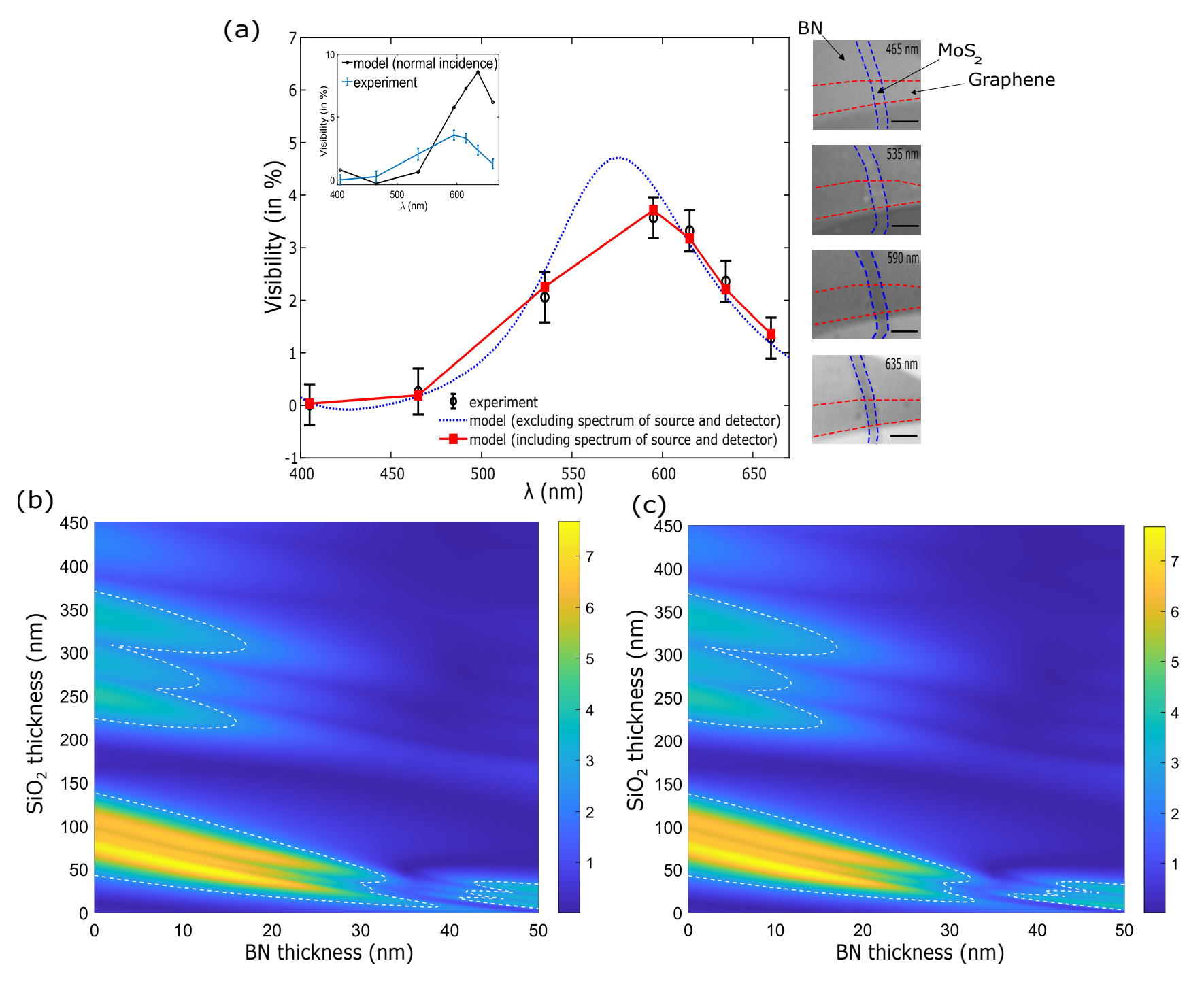

Figure S3. (a) Visibility as a function of wavelength for graphene-BN-MoS ${ }_{2}$ heterostructure showing experimental and theoretical plots. The panels to the right of (a) are optical micrographs under the illumination of LEDs of different wavelengths (namely $465 \mathrm{~nm}, 535 \mathrm{~nm}, 590 \mathrm{~nm}$ and $635 \mathrm{~nm}$ ) converted to greyscale. The scale bar at the bottom right is $2 \mu \mathrm{m}$. Color plots of the maximum of absolute visibilities of (b) graphene-BN-MoS 2 and (c) graphene- $\mathrm{MoS}_{2}-\mathrm{BN}$ heterostructures. White dashed lines are contours drawn at 2.5\% absolute visibility. 


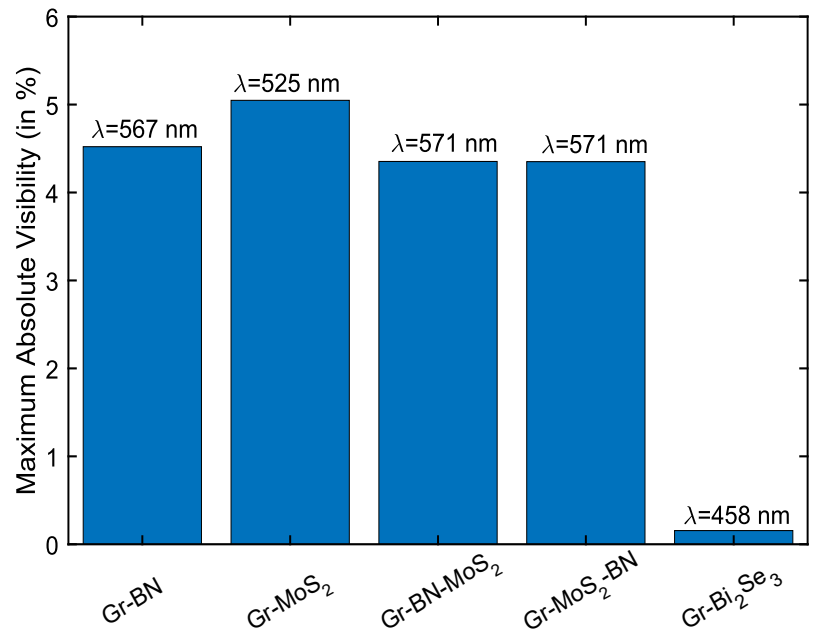

Figure S4. Maximum absolute visibilities and their peak wavelengths for various heterostructures. $\mathrm{BN}$ thickness is assumed to be $11 \mathrm{~nm}$ and $\mathrm{MoS}_{2}$ is assumed to be monolayer in all heterostructures. $\mathrm{Bi}_{2} \mathrm{Se}_{3}$ thickness is assumed to be $10 \mathrm{~nm}$. 
(a)


$\mathrm{BN}$

(b)



(c)



(e)
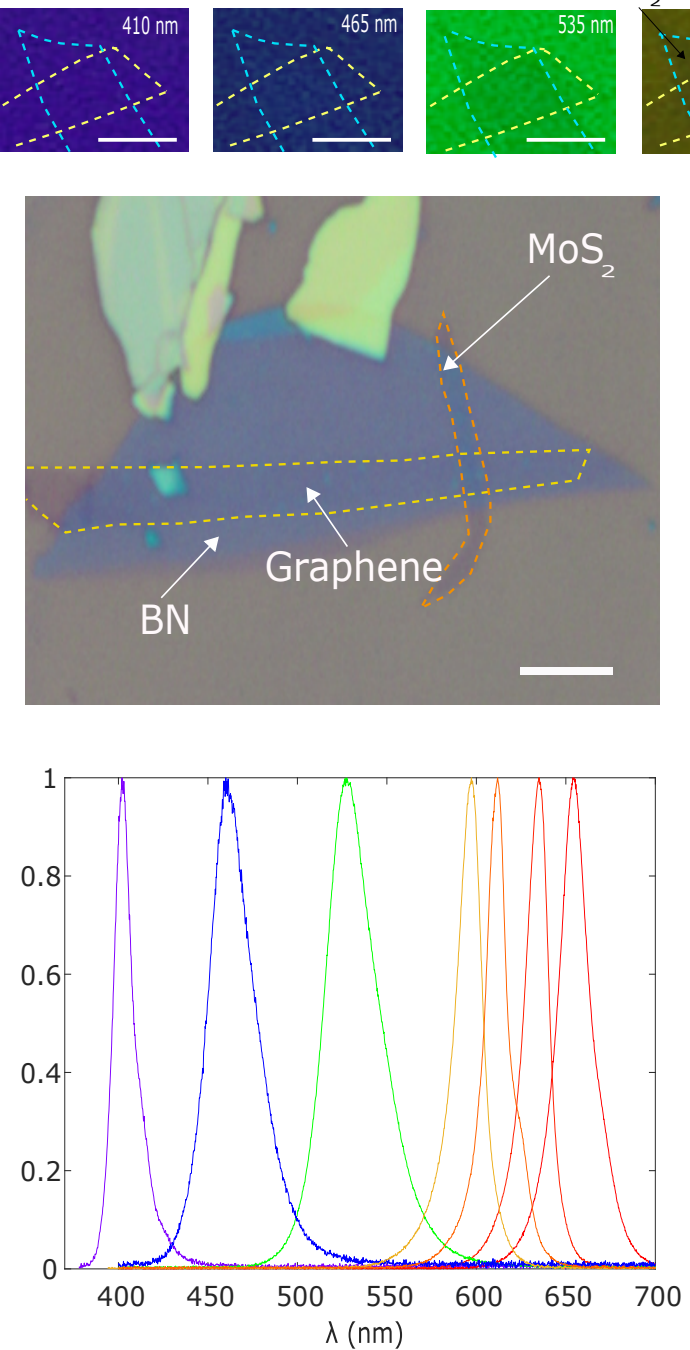

MoS Graphene


(d)



(f)

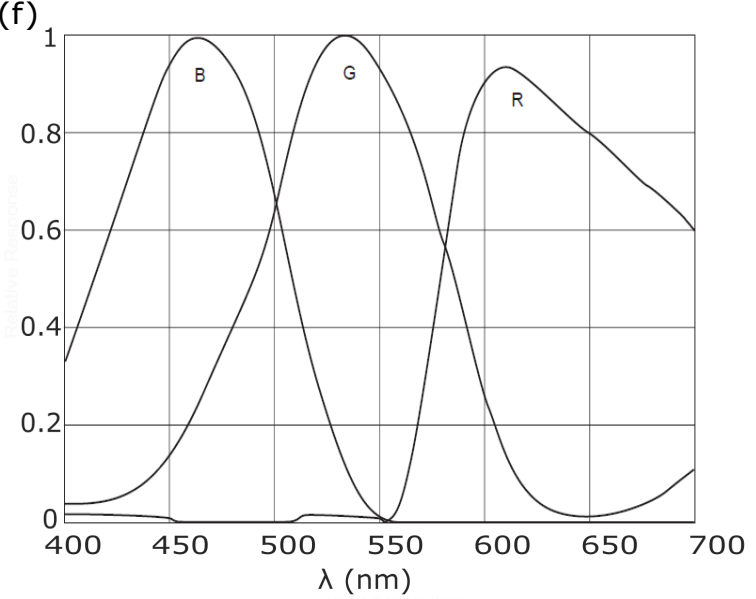

Figure S5. Images of (a) graphene-BN-MoS2 and (b) graphene-MoS 2 heterostructures illuminated by LEDs of different wavelengths. The scale bar at the bottom right of these two sets of images are $2 \mu \mathrm{m}$ in length. (c) Graphene-BN-MoS 2 and (d) graphene-MoS 2 heterostructures illuminated by a typical microscope light source. The scale bar at the bottom right of these two images are $5 \mu \mathrm{m}$ in length. (e) Spectrum of the LEDs and (f) spectral sensitivity of the camera used in the experiment. 



Figure S6. Color plots of maximum absolute visibilities of the three channels as a function of $\mathrm{SiO}_{2}$ thickness and BN thickness for (a) PMMA-graphene-BN and (c) BN-graphene-BN heterostructures. Color plots of maximum absolute visibilities of the three channels for (b) PMMA-graphene-MoS 2 heterostructure as a function of $\mathrm{SiO}_{2}$ thickness and number of $\mathrm{MoS}_{2}$ layers and (d) graphene$\mathrm{Bi}_{2} \mathrm{Se}_{3}$ heterostructure as a function of $\mathrm{SiO}_{2}$ thickness and $\mathrm{Bi}_{2} \mathrm{Se}_{3}$ thickness. Thickness of PMMA in (a) and (b) is assumed to be $380 \mathrm{~nm}$ (double layer of PMMA spin-coated at $5000 \mathrm{RPM}$ ) and in $\mathrm{BN}$-graphene-BN heterostructure, the thickness of the top BN is assumed to be $10 \mathrm{~nm}$. 



Figure S7. (a) Visibility of graphene- $\mathrm{ZrS}_{2}$ heterostructure as a function of wavelength of light for a fixed $\mathrm{SiO}_{2}$ thickness of $285 \mathrm{~nm}$. (b) Color plot of maximum absolute visibilities of the three channels for graphene- $\mathrm{ZrS}_{2}$ heterostructure as a function of $\mathrm{SiO}_{2}$ thickness and wavelength of light. Both graphene and $\mathrm{ZrS}_{2}$ are assumed to be monolayers. 